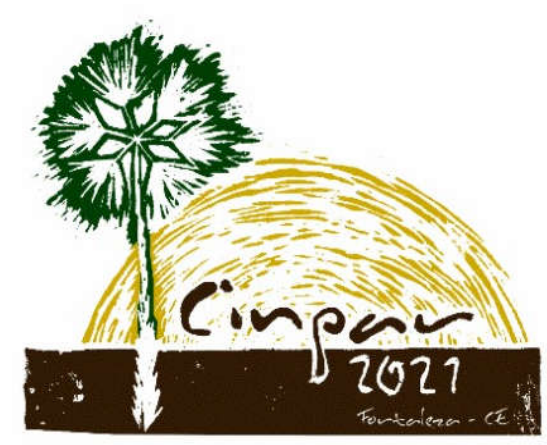

XVII Congresso Internacional sobre Patologia e
Reabilitação das Construções

XVII Congreso Internacional sobre Patología y Rehabilitación de las Construcciones

XVII International Conference on Pathology and Constructions Rehabilitation

FORTALEZA (Brasil), 3 a 5 de junho de 2021

https://doi.org/10.4322/CINPAR.2021.038

\title{
Tratamento de dados de ensaio ultrassônico em alvenarias: uma Revisão Sistemática de Literatura
}

\section{Data treatment from ultrasonic tests in masonry: a systematic literature review}

\author{
Estevão DAMASCENO FILHO ${ }^{1}$, Tálisson RODRIGUES ${ }^{2}$, Rosineide PAZ ${ }^{3}$, Esequiel MESQUITA ${ }^{3}$ \\ ${ }^{1}$ Mestrando em Engenharia Civil - UFC, Ceará, Brasil, damascenoestevao@alu.ufc.br \\ ${ }^{2}$ Graduando em Engenharia Civil - UFC, Ceará, Brasil, talisson.ecivil@alu.ufc.br \\ ${ }^{3}$ Profa. Drä. da UFC, Ceará, Brasil, rfdapaz@ufc.br \\ ${ }^{4}$ Prof. Dr. da UFC, Ceará, Brasil, emesquita@ufc.br
}

\begin{abstract}
Given their high cultural value, historic buildings play an important role for societies, so their conservation and preservation are the focus of several studies. From the Civil Engineering perspective, they represent a great challenge, as they show great constructive variability and refined care treatment regarding the application of tests, since these buildings have high historical value. In this context, non-destructive techniques appear as a tool for analyzing those structures in need of differentiated care. The ultrasound test, in particular, has been gaining prominence in the area due to its low cost and ease of reapplication. To better understand the scientific scenario, the present work aims to carry out a systematic review of the literature in the main databases to learn how to interpret ultrasonic data in solid masonry. It was found that the study of ultrasound application on masonry has increased in recent years, but not in regard to the use of statistical models for data interpretation, for which the amount of published works is still relatively low. Thus, a gap in literature was noticed, opening the space for the development of new research.
\end{abstract}

Keywords: Masonry. Data. Non-destructive test. Ultrasonic wave.

Resumo: Dado o seu elevado valor cultural, as construções históricas apresentam um importante papel para as sociedades, por isso sua conservação e preservação são enfoque de diversos estudos. Do ponto de vista da Engenharia Civil, elas representam um grande desafio, pois apresentam grande variabilidade construtiva e necessitam de cuidados refinados quanto à aplicação de ensaios, visto que essas edificações possuem elevado valor histórico. Nesse contexto, as técnicas não destrutivas surgem como uma ferramenta de análise dessas estruturas que precisam de cuidados diferenciados. O ensaio de ultrassom, em especial, vem ganhando destaque na área por seu baixo custo e facilidade de reaplicação. Para entender melhor o cenário científico, o presente trabalho tem como objetivo a realização de uma revisão sistemática de literatura nas principais bases de dados para conhecer como se dão as interpretações dos dados ultrassônicos em alvenarias maciças. Constatou-se que o estudo da aplicação de ultrassom em alvenaria cresceu nos últimos anos, mas no tocante à utilização de modelos estatísticos para interpretação dos dados, ainda é relativamente baixa em relação a quantidade de trabalhos publicados. Assim, uma lacuna a ser preenchida foi observada, abrindo espaço para o desenvolvimento de novas pesquisas.

Palavras-chave: Alvenaria. Dados. Ensaio Não Destrutivo. Onda Ultrassônica. 


\section{Introdução}

Para a realização de planos de manutenção ou de reabilitação de uma edificação, um dos principais pontos é avaliar as condições em que as estruturas se encontram, de forma que, para isso, é necessário ter o conhecimento de variáveis reais, como a geometria e a resistência dos elementos (CASCANTE et al., 2008). Ainda segundo Cascante et al. (2008) , as informações a respeito destas variáveis são obtidas comumente através de ensaios destrutivos, que geralmente são caros e passíveis de não serem realizados.

Os ensaios destrutivos são bastante aceitos em função da precisão de seus resultados, porém é necessário retirar amostras do objeto em análise que, por sua vez, são destruídas para a obtenção dos parâmetros mecânicos (ZIELIŃSKA; RUCKA, 2018). Contudo, os END (Ensaios Não Destrutivos) vêm se desenvolvendo e proporcionando uma coleta detalhada e rápida de informações das estruturas, com as vantagens de reduzir tempo e custos, preservar a estética das edificações, possibilitar a realização in situ com a capacidade de repetição das medições, e, assim, ser um dos métodos mais empregados no campo do patrimônio cultural (AHMAD et al., 2009; MISHRA et al., 2019; NOOR-E-KHUDA; ALBERMANI, 2019; SCHABOWICZ, 2019).

Com relação aos END voltados para estruturas de alvenaria, o método ultrassônico tem sido bem empregado, principalmente em função do baixo custo e da facilidade de operação (KHODAVERDIAN; SHOMALI, 2010). O que contribui para a sua aplicação é sua capacidade de qualificar as alvenarias através da morfologia da seção das paredes, detectar a existência de vazios e falhas, bem como encontrar padrões de trincas e danos (BINDA et al., 2003). Isso é possível pois qualquer obstáculo ou até mesmo mudança de material no caminho da propagação da onda provoca alteração no seu tempo de viagem (ZIELIŃSKA; RUCKA, 2018).

Devido a importância que os ensaios não destrutivos apresentam ao longo do tempo, estes se tornaram alvos de trabalhos que os abordam em forma de revisão, tanto para mostrar quais ensaios existem, como para a evolução dos mesmos e das pesquisas em que foram empregados. Moropoulou e Labropoulos (2015) apresentam as capacidades dos ensaios não destrutivos em patrimônios históricos e como essas técnicas auxiliam na tomada de decisão em relação a um processo de restauração, manutenção ou monitorização. Tuğla et al. (2018) apresentam os danos mais comuns que ocorrem em construções históricas e as suas principais causas.

Apesar de o ultrassom ser um dos métodos mais empregados para alvenarias, principalmente de pedra, notou-se na literatura uma lacuna relacionada aos trabalhos que envolvem o ensaio de ultrassom voltado para alvenarias de tijolo maciço, assim como a maneira em que os dados são tratados a fim de obter as respostas buscadas na pesquisa. Dessa forma, o presente capítulo buscou, por meio da Revisão Sistemática de Literatura, conhecer as pesquisas já realizadas e em andamento sobre o uso do ensaio de ultrassom e como os dados obtidos estão sendo tratados para caracterizar e analisar as alvenarias de tijolo maciço, que são características das construções históricas brasileiras, de forma a observar lacunas que possam ser exploradas em trabalhos futuros.

\section{Princípios básicos dos métodos ultrassônicos}

\subsection{Propagação da onda ultrassônica}

As ondas ultrassônicas são ondas sonoras mecânicas superiores ao intervalo audível para o ser humano, que variam entre $20 \mathrm{~Hz}$ a $20000 \mathrm{~Hz}$. Segundo Silva Neto (2014), essas ondas são originadas da deformação de um meio elástico e, para se propagarem, necessitam de um meio material, por isso não se propagam no vácuo.

Ainda segundo o já referido autor, a onda se propaga através de quaisquer forças externas, causando o deslocamento subatômico de um elemento que, por sua vez, desloca o elemento adjacente promovendo um desenvolvimento infinitesimal da onda. Esta propagação é bastante sensível ao meio, sendo o seu tempo de percurso afetado pelo que for encontrado no caminho e assim se torna possível a determinação do estado do material.

Uma característica importante que permite que o uso de ondas ultrassônicas seja tão empregado na área da engenharia civil é o fato que qualquer alteração nas características do material influencia na transmissão da onda. O sinal recebido no transdutor receptor consegue transmitir informações sobre o material e a 
estrutura, como também características, por exemplo, densidade, porosidade, rigidez, entre outros (MEOLA et al., 2005).

\subsection{Método ultrassônico}

Através do uso da propagação de ondas, o ensaio de velocidade do pulso ultrassônico é bastante aplicado na caracterização de estruturas, destacando-se entre os ensaios não destrutivos, como comentado em Antonaci et al. (2013). Esta técnica consiste na emissão de um pulso ultrassônico produzido através de um cristal piezoelétrico dentro dos transdutores do equipamento. Este cristal, segundo Khairi et al. (2019), trabalha como um conversor de energia elétrica em energia mecânica produzindo a onda que, ao chegar no outro transdutor, se transforma em pulso elétrico pelo processo inverso e a partir desta segunda recepção se processa o tempo.

A aplicação do ensaio se dá através de três configurações de posicionamentos diferentes, que são os métodos direto, indireto e semidireto. As normalizações que aplicam o método ultrassônico, por exemplo as normas NBR 8802 (ABNT, 2019), ASTM C 597 (2016) e BS EM 12504-4 (2004) para concreto, e recentemente NBR 16805 (ABNT, 2020) para alvenarias, falam do uso destes com relação a como se encontra o objeto de análise e qual a aplicação requerida. Para maior precisão e com as duas faces opostas acessíveis é aplicado o método direto. Quando se tem duas faces para ensaio mas elas não são opostas usa-se o método semidireto e, quando apenas um lado está acessível, a opção é o método indireto.

\section{Metodologia}

Com o propósito de entender melhor o que já foi estudado sobre como os dados de pulso ultrassônico estão sendo tratados estatisticamente, foi realizada uma Revisão Sistemática de Literatura (RSL), conhecida em inglês como Systematic Literature Review (SRL) com auxílio do programa State of the Art through Systematic Review (START) em três plataformas: Science Direct, Scopus e Web of Science.

Seguindo as orientações referentes a uma RSL, foram selecionados a partir das bases de dados 942 artigos, nacionais e internacionais, publicados a partir de 1991 até o ano de 2019. As palavras-chave determinadas para realizar as buscas foram data, masonry, non-destructive e ultrasonic. Do total de artigos selecionados, 35 foram aceitos e os demais rejeitados, seja por abordagem fora do tema buscado ou por serem duplicados. Após a etapa de seleção, os artigos foram avaliados de forma mais criteriosa na etapa de extração, onde dos 35 artigos selecionados, 13 foram aceitos e qualificados nesta etapa (Figura 1).

Como sugerido pelo START, ao final da etapa de extração foi realizada uma classificação qualitativa de ordem de prioridade dos artigos. Estes foram divididos em três categorias de prioridade, sendo: "Muito Alta" para os artigos que utilizaram métodos estatísticos para tratamento dos dados obtidos a partir de ensaio de ultrassom realizados em alvenarias de tijolo maciço; quando fizeram a aplicação do ultrassom com outros métodos não destrutivos também em alvenarias ficaria classificado como prioridade "Alta"; e quando os dados foram apenas utilizados para realizar correlações foi categorizado como prioridade "Baixa".

Foram encontradas algumas dificuldades quanto ao levantamento dos artigos no tocante ao uso das palavras chaves em relação ao emprego do ensaio de ultrassom, pois a aplicação do ensaio pode ser abordada por variadas denominações. Outro fator a ser relatado sobre a RSL é que a maioria das pesquisas que empregam ensaio de ultrassom são voltadas para concreto que, em função da presença das palavras-chave no corpo do artigo, foram captados na busca e resultaram em um grande número de artigos rejeitados. 


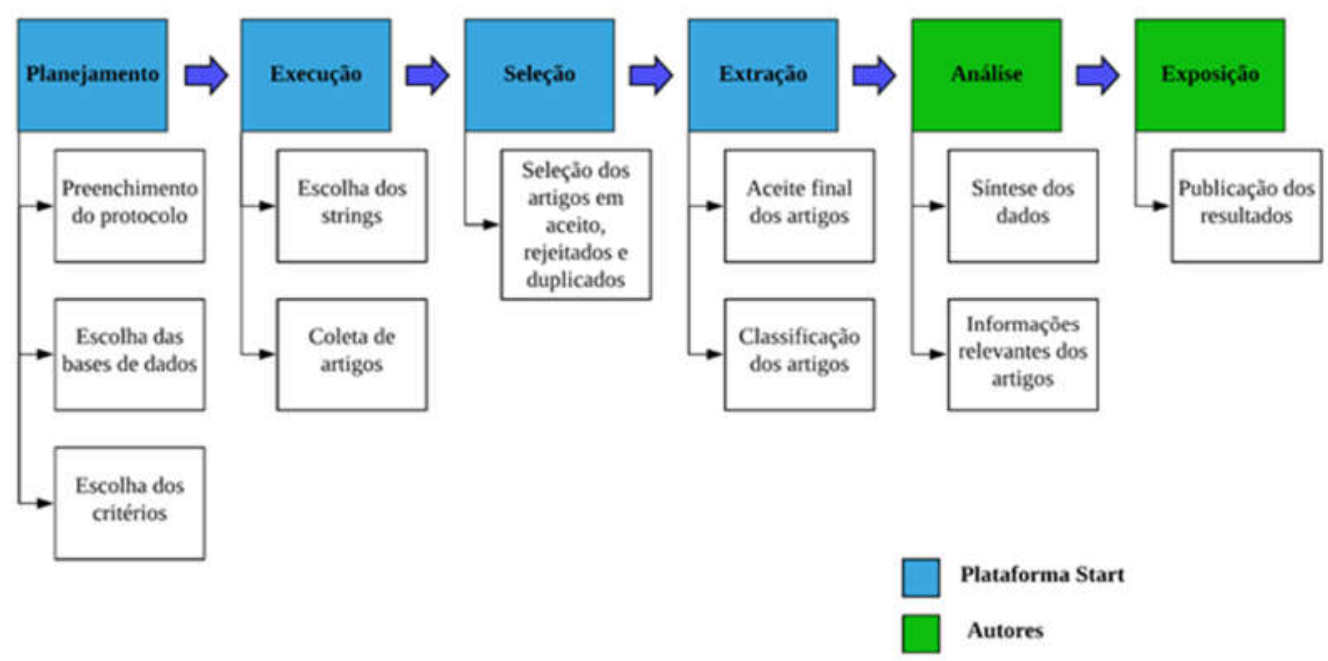

Figura 1 - Delineamento da Revisão Sistemática de Literatura

\section{Resultados e discussões}

A revisão sistemática mostrou o aumento crescente no número de publicações nos dois últimos anos, concentrando aproximadamente $50 \%$ das publicações (Figura 2). Isso mostra que grupos de pesquisa estão desenvolvendo ou buscando explorar mais o uso de ensaios não destrutivos em alvenarias de tijolo maciço como um todo ou de seus elementos, de forma a entender seu comportamento, utilizando ensaios ultrassônicos. Entre as universidades envolvidas nas publicações pode-se citar "Polytechnic University of Bari", "Universitat Politècnica de Catalunya", 'Universita' di Napoli Federico II", Universidade do Porto, Universidade de Aveiro, Universidade Vale do Acaraú, "Politecnico di Torino", entre outras.

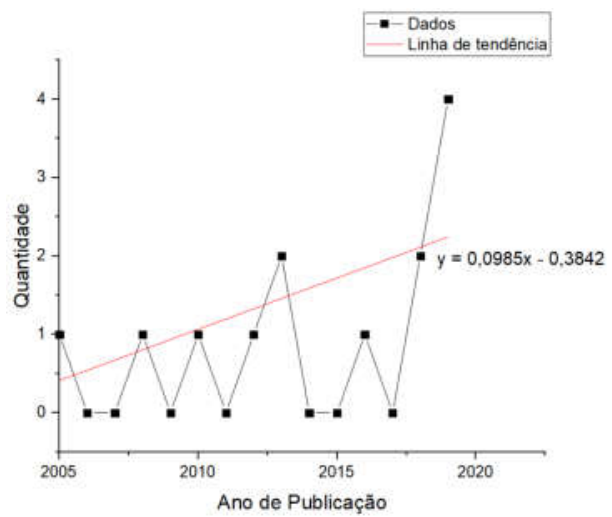

Figura 2 - Gráfico do quantitativo de artigos extraídos por ano de publicação

Como visto anteriormente, há três formas de realizar o ensaio de ultrassom, através dos métodos direto, semidireto e indireto. Assim, foi observado em que situações cada modo de aplicação foi empregado. A aplicação da técnica de posicionamento do ultrassom direto está pontualmente ligado ao viés das pesquisas levantadas que, em sua maioria, realizaram o ensaio em ambiente controlado com um componente isolado da alvenaria, como visto em Mishra et al. (2019), ou ainda em uma alvenaria de pequeno porte onde os dois lados da amostra eram facilmente acessíveis como visto em Zielińska e Rucka (2018) e Khodaverdian e Shomali (2010). Além disso, houve aplicação em componentes da alvenaria como tijolos ou argamassas, como observado em Makoond et al. (2019) e Noor-e-Khuda e Albermani (2019).

A parcela de artigos que usaram o método indireto de medição teve como direcionamento de pesquisa a análise de estruturas em campo, como visto em Camassa et al. (2019) e Mesquita et al. (2018). Já trabalhos como (MEOLA et al., 2005) e (FAELLA et al., 2012) aplicaram dois modos de ensaio, sendo eles direto e 
indireto. Além disso, outros trabalhos utilizaram-se da técnica de tomografia ultrassônica a fim de obter uma representação da estrutura interna das estruturas, como abordado em Khodaverdian e Shomali (2010), em Zielińska e Rucka (2018)) e Cotic et al. (2013).

Observou-se que, dentre os artigos analisados, 53,85\% apresentaram o uso de mais de uma técnica não destrutiva em até 4 métodos diferentes, como por exemplo, teste de radar penetrante em superfície, análise de termografia infravermelha, inspeção por fibroscópio, entre outros . Os outros artigos apenas aplicaram como ensaio não destrutivo o ultrassom. Dessa forma, a utilização de mais de um método não destrutivo se deu em laboratório, fazendo-se uso das especificidades de cada técnica para melhor caracterização e compreensão das alvenarias e seus componente ou então em trabalhos de campo, com uma análise integrada de uma edificação como um todo, caso da pesquisa de Faella et al. (2012).

Na literatura é recorrente a preocupação com as características mecânicas dos componentes, pois estas propriedades asseguram a estabilidade e segurança da estrutura, como visto em Mishra et al. (2019). Neste trabalho, a resistência à compressão em tijolos, argamassa e paredes de alvenaria é prevista por meio da combinação de resultados dos ensaios de martelo de Schmidt e ultrassom; a particularidade deste estudo vem da utilização de um modelo de regressão linear, combinado com uma rede neural artificial (ANFIS) e um sistema de inferência neuro-fuzzy que influenciaram para a obtenção de resultados mais precisos e bem próximos dos obtidos experimentalmente.

Em um estudo desenvolvido por Antonaci et al. (2013), que em função do aumento da umidade e da presença de sais solúveis, que podem causar danos às edificações, foi verificado a eficiência de uma técnica desenvolvida pelos autores chamada de Scaling Subtraction Method (SSM). Esta técnica se baseia em uma técnica ultrassônica não linear, voltada para caracterizar danos decorrentes da cristalização de sais expansivos em alvenarias. Segundo os autores, foi observada uma lacuna no meio técnico sobre as potencialidades de técnicas ultrassônicas não lineares para alvenarias.

Em termos de caracterizar as estruturas, Paasche et al. (2008), aplicaram uma técnica chamada de inversão cooperativa zonal com o fim de desenvolver um método para detectar vazios a partir da coleta dos dados de tempos de viagem ultrassônicos e georadar. Entre algumas observações percebidas ao longo do estudo, foi visto que a velocidade da onda diminuía na alvenaria quando ia em direção às bordas. Eles fizeram uma análise do modelo com as técnicas separadas e depois combinadas, concluindo que a inversão cooperativa zonal permitiu um melhor delineamento tanto do tamanho como da posição das cavidades.

Avanços na aplicação do método indireto em alvenarias foi apresentado por Mesquita et al. (2018), em que foram realizados ensaios de ultrassom em uma igreja histórica do século XVIII localizada na cidade de Aracati - Ceará, Brasil. A principal contribuição foi o desenvolvimento de uma forma sistemática para coletar as medidas das medições ultrassônicas de forma indireta, haja vista a variabilidade do modo de medição em outras pesquisas. Ao final do processamento são elaborados mapas de contorno que permitem a comparação entre os painéis avaliados.

O sistema de medição desse trabalho contribuiu para a formulação da norma brasileira pela ABNT, nomeadamente a NBR 16805: Ensaios não destrutivos - Ultrassom - Caracterização de painéis por velocidade de propagação de onda ultrassônica (ABNT, 2020). A publicação dessa norma brasileira mostra um avanço nessa área no Brasil, haja vista que até a publicação desta norma os ensaios de caracterização ultrassônica utilizavam como base a NBR 8802 (ABNT, 2019), em que é aplicada a determinação da velocidade de propagação da onda ultrassônica para o concreto endurecido.

Quanto às pesquisas relacionadas ao tema em análise aplicadas aqui no Brasil, foram poucos os estudos e, muitas vezes, não apresentavam um tratamento estatístico mais robusto. Assim como em alguns estudos internacionais, em Souza et al. (2014), e também em Melo (2014), foram empregados os ensaios de determinação da velocidade de propagação ultrassônica e escloremetria juntamente com o ensaio destrutivo de resistência à compressão, de forma a criar uma correlação para estimar a resistência de paredes de tijolo cerâmico e blocos cimentícios. Por sua vez, em Rubens et al. (2019), foram realizadas correlações entre a velocidade de propagação ultrassônica e a resistência à compressão de tijolos cerâmicos maciços de forma a caracterizar esse tipo de material empregado em alvenarias portantes de edificações do século XX.

Assim, percebe-se nos trabalhos o uso dos dados ultrassônicos para estabelecimento de correlações relacionadas a parâmetros mecânicos, como resistência à compressão, módulo de elasticidade, entre outros.

Tratamento de dados de ensaio ultrassônico em alvenarias: uma Revisão Sistemática de Literatura 
No entanto, nos últimos anos, além das estimativas, buscam-se modelos e técnicas que, por meio de um tratamento maior dos dados, possam caracterizar as alvenarias.

Atualmente, há inúmeros testes não destrutivos que podem ser aplicados para as mais variadas finalidades, seja detecção de vazios, falhas, fissuras, entre outras. Apesar da grande quantidade de testes, muitas vezes estes têm que ser combinados, porque quando utilizados isoladamente ainda não são capazes de apresentar um resultado confiável. A questão que fica é: com todo esse avanço, as pesquisas deveriam tentar focar cada vez mais em desenvolver cada método até atingir o seu potencial, já que alguns são mais baratos do que outros, ou investir no uso combinado das técnicas?

A ideia é buscar desenvolver cada vez mais pesquisas relacionadas a ensaios de ultrassom, tentando explorar ao máximo o seu uso, principalmente quando se fala do método indireto, visto que, segundo outras pesquisas e estudos (BOSILJKOV et al., 2010; GUADAGNUOLO et al., 2014; LIGNOLA; MANFREDI, 2010), este é menos preciso que o método direto. Porém, como comentado por Meola et al. (2005), o método indireto é o mais adequado para analisar a qualidade de camadas mais rasas, caso o objetivo seja analisar o revestimento, como também para a aplicação em alvenarias que, muitas vezes, é o método que pode ser aplicado quando se trata de ultrassom.

\section{Conclusões}

De acordo com as bases de dados pesquisadas na RSL, percebeu-se que o estudo do ultrassom está, até então, muito relacionado às estruturas de concreto e, quando se trata a alvenarias, a atenção é voltada para alvenarias de pedra. Mas também pode-se perceber com a revisão que a partir de meados de 2017 houve uma crescente de publicações acerca de estudos em alvenarias de tijolo maciço. Observou-se que o uso de modelos estatísticos para análise dos dados ainda representa uma lacuna por aparecer em poucos trabalhos.

Como contribuição da RSL para a comunidade científica se tem as evidências de um crescimento importante dos estudos das alvenarias e do ultrassom como ferramenta de destaque que pode contribuir diretamente na preservação do patrimônio histórico por meio dos ensaios. Porém, o uso de modelos estatísticos para análise dos dados ainda representa uma lacuna por aparecer em poucos trabalhos e dado a importância de se garantir a fiabilidade das interpretações para melhores resultados.

\section{Agradecimentos}

À CAPES, pelo apoio financeiro às bolsas de mestrado e auxílio do projeto de $n^{\circ} 7623$ do PIBIC cadastrado na plataforma Ícaro Moreira.

\section{Referências Bibliográficas}

AMERICAN SOCIETY FOR TESTING AND MATERIALS. ASTM C 597-16. Standard test method for pulse velocity through concrete. Pensilvânia, 2016.

ASSOCIAÇÃO BRASILEIRA DE NORMAS TÉCNICAS. NBR 16805: Ensaios não destrutivos - Ultrassom Caracterização de painéis por velocidade de propagação ultrassônica. 1 ed. Rio de Janeiro: ABNT, 2020. 11 p. ASSOCIAÇÃO BRASILEIRA DE NORMAS TÉCNICAS. NBR 8802. Concreto endurecido - Determinação da velocidade de propagação de onda ultrassônica. no. 153, p. 114-119, 2019.

AHMAD, A.; PAMPLONA, M.; SIMON, S. Ultrasonic testing for the investigation and characterization of stone - a non-destructive and transportable tool. Studies in Conservation, vol. 54, no. sup1, p. 43-53, 2009. https://doi.org/10.1179/sic.2009.54.supplement-1.43.

ANTONACI, P.; FORMIA, A.; GLIOZZI, A. S.; SCALERANDI, M.; TULLIANI, J. M. Diagnostic application of nonlinear ultrasonics to characterize degradation by expansive salts in masonry systems. NDT and $\mathbf{E}$ International, vol. 55, p. 57-63, 2013. DOI 10.1016/j.ndteint.2013.01.013. Available at: http://dx.doi.org/10.1016/j.ndteint.2013.01.013. 
BINDA, Luigia; SAISI, A.; ZANZI, L. Sonic tomography and flat-jack tests as complementary investigation procedures for the stone pillars of the temple of S. Nicolò 1'Arena (Italy). NDT and E International, vol. 36, no. 4, p. 215-227, 2003. https://doi.org/10.1016/S0963-8695(02)00066-X.

BOSILJKOV, Vlatko; URANJEK, Mojmir; ŽARNIĆ, Roko; BOKAN-BOSILKOV, Violeta. An integrated diagnostic approach for the assessment of historic masonry structures. Journal of Cultural Heritage, vol. 11, no. 3, p. 239-249, 2010. https://doi.org/10.1016/j.culher.2009.11.007.

BRITISH STANDARD. BS EN 12504: Part 4. Determination of ultrasonic pulse velocity London, British Standars Institution. 2004.

CAMASSA, D.; CASTELLANO, A.; FRADDOSIO, A.; PICCIONI, M. D. Improvements of the Ultrasonic Tomography for Applications to Historical Masonry Constructions. [S. I.]: Springer International Publishing, 2019. vol. 18, . DOI 10.1007/978-3-319-99441-3. Available at: http://link.springer.com/10.1007/978-3-31999441-3.

CASCANTE, G.; NAJJARAN, H.; CRESPI, P. Novel methodology for nondestructive evaluation of brick walls: Fuzzy logic analysis of MASW tests. Journal of Infrastructure Systems, vol. 14, no. 2, p. 117-128, 2008. https://doi.org/10.1061/(ASCE)1076-0342(2008)14:2(117).

COTIC, P.; JAGLICIC, Z.; NIEDERLEITHINGER, E.; EFFNER, U.; KRUSCHWITZ, S.; TRELA, C.; BOSILJKOV, V. Effect of moisture on the reliability of void detection in brickwork masonry using radar, ultrasonic and complex resistivity tomography. Materials and Structures/Materiaux et Constructions, vol. 46, no. 10, p. 1723-1735, 2013. https://doi.org/10.1617/s11527-012-0011-3.

FAELLA, G.; FRUNZIO, G.; GUADAGNUOLO, M.; DONADIO, A.; FERRI, L. The Church of the Nativity in Bethlehem: Non-destructive tests for the structural knowledge. Journal of Cultural Heritage, vol. 13, no. 4 SUPPL., p. e27-e41, 2012. DOI 10.1016/j.culher.2012.10.014. Available at: http://dx.doi.org/10.1016/j.culher.2012.10.014.

GUADAGNUOLO, Mariateresa; FAELLA, Giuseppe; DONADIO, Alfonso; FERRI, Luca. Integrated evaluation of the Church of S. Nicola di Mira: Conservation versus safety. NDT and E International, vol. 68, p. 53-65, 2014. DOI 10.1016/j.ndteint.2014.08.002. Available at: http://dx.doi.org/10.1016/j.ndteint.2014.08.002.

KHAIRI, Mohd Taufiq Mohd; IBRAHIM, Sallehuddin; YUNUS, Mohd Amri Md; FARAMARZI, M.; SEAN, Goh Pei; PUSPPANATHAN, Jaysuman; ABID, A. Ultrasound computed tomography for material inspection: Principles, design and applications. Measurement: Journal of the International Measurement Confederation, vol. 146, p. 490-523, 2019. DOI 10.1016/j.measurement.2019.06.053. Available at: https://doi.org/10.1016/j.measurement.2019.06.053.

KHODAVERDIAN, A.; SHOMALI, B. The actions of axial loads on masonries - Ultrasonic verifications and statistical analysis of obtained data. Large Structures and Infrastructures for Environmentally Constrained and Urbanised Areas, , p. 886-887, 2010. https://doi.org/10.2749/222137810796064246.

LIGNOLA, Gian Piero; MANFREDI, Gaetano. A combination of NDT methods for the restoration of monumental façades: The case study of Monte di Pietà (Naples, Italy). Journal of Cultural Heritage, vol. 11, no. 3 , p. 360-364, 2010. DOI 10.1016/j.culher.2009.11.010. Available at: http://dx.doi.org/10.1016/j.culher.2009.11.010.

MAKOOND, N.; PELÀ, L.; MOLINS, C. Dynamic elastic properties of brick masonry constituents. Construction and Building Materials, vol. 199, p. 756-770, 2019. DOI 10.1016/j.conbuildmat.2018.12.071. Available at: https://doi.org/10.1016/j.conbuildmat.2018.12.071.

MELO, Aroldo Vieira. Estudo da resistência à compressão em alvenarias pelos métodos não destrutivos. Revista Pernambucana de Tecnologia, Recife, v. 2, n. 2, p. 68-81, abr. 2014.

MEOLA, C.; DI MAIO, R.; ROBERTI, N.; CARLOMAGNO, G. M. Application of infrared thermography and geophysical methods for defect detection in architectural structures. Engineering Failure Analysis, vol. 12, no. 6 SPEC. ISS., p. 875-892, 2005. https://doi.org/10.1016/j.engfailanal.2004.12.030.

MESQUITA, E.; MARTINI, R.; ALVES, A.; MOTA, L.; RUBENS, T.; ANTUNES, P.; VARUM, H. Heterogeneity detection of Portuguese-Brazilian masonries through ultrasonic velocities measurements. Journal of Civil Structural Health Monitoring, vol. 8, no. 5, p. 847-856, 2018. https://doi.org/10.1007/s13349-018-0312-5. 
MISHRA, M.; BHATIA, A. S.; MAITY, D. A comparative study of regression, neural network and neuro-fuzzy inference system for determining the compressive strength of brick-mortar masonry by fusing nondestructive testing data. Engineering with Computers, no. 0123456789, 2019. DOI 10.1007/s00366-01900810-4. Available at: https://doi.org/10.1007/s00366-019-00810-4.

MOROPOULOU, A. I.; LABROPOULOS, K. C. Non-destructive testing for assessing structural damage and

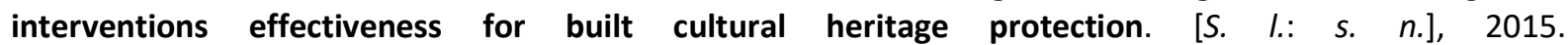
https://doi.org/10.4018/978-1-4666-8286-3.ch015.

NOOR-E-KHUDA, S.; ALBERMANI, F. Mechanical properties of clay masonry units: Destructive and ultrasonic testing. Construction and Building Materials, vol. 219, p. 111-120, 2019. DOI 10.1016/j.conbuildmat.2019.05.166. Available at: https://doi.org/10.1016/j.conbuildmat.2019.05.166.

PAASCHE, H.; WENDRICH, A.; TRONICKE, J.; TRELA, C. Detecting voids in masonry by cooperatively inverting P-wave and georadar traveltimes. Journal of Geophysics and Engineering, vol. 5, no. 3, p. 256-267, 2008. https://doi.org/10.1088/1742-2132/5/3/002.

RUBENS, T.; OLIVEIRA, F. L. De; MESQUITA, E. Caracterização de tijolos históricos luso-brasileiros pelo método ultrassônico. no. February 2020, 2019. .

SCHABOWICZ, K. Non-destructive testing of materials in civil engineering. Materials, vol. 12, no. 19, p. 1-13, 2019. https://doi.org/10.3390/ma12193237.

SILVA NETO, R. J. da. AVALIAÇÃO DE TÉCNICA DE MEDIDA DO TEMPO DE PERCURSO DE ONDAS ULTRASSÔNICAS BASEADO NO ESPECTRO DE FREQÜÊNCIA. 2014. 2014.

SOUZA, L. G. M. S. et al. Estudo da resistência à compressão em alvenarias pelos métodos não destrutivos. In: CONGRESSO BRASILEIRO DE ENGENHARIA E ClêNCIA DOS MATERIAIS, 21., 2014, Cuiabá. Anais. p. 917924.

TUĞLA, R.; DEMIRCAN, R. K.; KAPLAN, G. A study on the use of advanced nondestructive testing methods on histroric structures. Lecture Notes in Civil Engineering, vol. 7, no. Isbs 2017, p. 536-549, 2018. https://doi.org/10.1007/978-3-319-64349-6_43.

ZIELIŃSKA, M.; RUCKA, M. Non-destructive assessment of masonry pillars using ultrasonic tomography. Materials, vol. 11, no. 12, p. 1-16, 2018. https://doi.org/10.3390/ma11122543. 